\title{
El cirujano de pie y tobillo y su rol en la era del COVID-19
}

\author{
The foot and ankle surgeon and his role in the COVID-19 era
}

\author{
Mariana Salazar del Villar, ${ }^{\star}$ Israel Gutiérrez Mendoza ${ }^{\ddagger}$

\begin{abstract}
* Médico adscrito al Servicio de Ortopedia del Hospital Central y Profesor adjunto de la Especialidad de Ortopedia y Traumatología del Hospital Central «Dr. Ignacio Morones Prieto», Universidad Autónoma de San Luis Potosí, UASLP. ¥ Ortopedista con Maestría en Ciencias Médicas. Presidente del Colegio de Ortopedia y Traumatología de Aguascalientes, A.C. Miembro del Comité Científico y Editorial FEMECOT.
\end{abstract}

Correspondencia: Dra. Mariana Salazar del Villar. Agustín Vera No. 265, Col. Tequisquiapan, 78250, San Luis Potosí, México. Correo electrónico: dramarianasalazar@live.com.mx

\section{RESUMEN}

Desde diciembre de 2019 se descubrió un nuevo virus en la provincia de Wuhan, China: el virus del SARS-CoV-2, el cual es altamente contagioso y tiene una rápida expansión a través de todo el mundo; debido a ello han seguido presentándose nuevos casos de la enfermedad que requieren ciertas recomendaciones en su atención médica durante el periodo de la pandemia. En relación a la consulta externa de pacientes con patología del pie y tobillo deberá realizarse un discernimiento entre las consultas que pueden aplazarse y las urgentes. En el caso de que no sea urgente se recomienda consultarlos a distancia mediante videoconferencia. En los pacientes que tenían cirugía programada durante el periodo de pandemia, se deberá valorar su diferimiento hasta terminar la contingencia sanitaria con excepción de la cirugía oncológica o aquélla que, en caso de demorarse ocasionaría secuelas. Para los pacientes con trauma agudo como fracturas expuestas, síndrome compartamental, pacientes con infección de heridas y pie diabético complicado deberán atenderse quirúrgicamente a pesar de la contingencia sanitaria. Se debe considerar la intervención quirúrgica de pacientes durante la contingencia sanitaria con un alto riesgo de adquirir la

\section{ABSTRACT}

Since December of 2019 there has been the discovery of a new virus in the province of Wuhan, China: the SARS-CoV2 virus, which is highly contagious and has had a fast expansion all across the globe; due to this there have been presenting new cases of the disease requiring certain recommendations in the medical attention during this time of pandemic. In regard to the outpatient appointments of patients with feet and ankle pathology, there should be a discerning of the appointments that can be postponed and those that are emergencies. In the case that they are not emergencies it is recommended to carry out the appointment from a distance by videoconference. For those patients who had an appointed surgery during this pandemic period, it should be assessed it's deferral until the end of the health contingency with the exception of an oncologic surgery or a case in which a delay of the surgery would cause health repercussions. For those with acute trauma like exposed fractures, compartment syndrome, patients with infected wounds and complicated diabetic foot disease will have to be attended surgically in spite of the health contingency. A surgical intervention during the health contingency should be considered 
enfermedad por parte del personal de salud durante dicho procedimiento, motivo por el cual al paciente se le debe realizar la prueba diagnóstica para el virus antes de la cirugía en caso de que sus condiciones lo permitan o considerar operarlo y esperar el resultado con posterioridad tomando las debidas precauciones de protección para el personal de salud mencionadas en las mejores guías de práctica clínica para cirujanos ortopedistas basadas en la evidencia COVID-19.

Palabras clave: COVID-19, cirugía, tratamiento, pie y tobillo, traumatología. with a high risk of acquiring the disease by the health personnel during the procedure, the reason for which the diagnosis test for the virus should be performed to the patient before the surgery if their condition allows it or it's considered operating them and wait for the results afterwards taking the due precautions of protection for the health personnel mentioned in the best guides of clinical practice for orthopedic surgeons based on evidence for the COVID-19.

Keywords: COVID-19, surgery, treatment, foot and ankle, traumatology.

\section{INTRODUCCIÓN}

En diciembre de 2019 se informó de una serie de casos de neumonía de etiología desconocida, las autoridades chinas identificaron como agente causante del brote un nuevo tipo de virus de la familia de los Coronaviridae, denominado SARS-CoV-2. El virus se introdujo en las personas a través de un reservorio animal no identificado, produciendo posteriormente una propagación de una persona a otra. ${ }^{1-3}$

Las infecciones en humanos provocan una amplia presentación clínica que va desde infecciones leves del tracto respiratorio superior hasta el Síndrome de Distrés Respiratorio Agudo (SDRA), sepsis, síntomas gastrointestinales, alteraciones dérmicas. El periodo de incubación es de 5.2 a 12.5 días, aunque se han descrito casos hasta de 24 días de incubación. La transmisión se produce por gotas respiratorias (hasta $2 \mathrm{~m}$ ) y por contacto de material contaminado en mucosas (oral, ocular y nasal), también se puede transmitir por aerosoles producidos durante algún procedimiento terapéutico. ${ }^{2,4}$

Para su diagnóstico se realizan pruebas moleculares (PCR) y secuenciación genómica, obteniendo muestras de los pacientes del tracto respiratorio superior e inferior. No existe un tratamiento específico, por lo que los aspectos fundamentales del tratamiento son soporte y manejo de las complicaciones, soporte temprano en el compromiso respiratorio, soporte respiratorio avanzado, manejo del shock séptico y tratamiento antimicrobiano. ${ }^{1,3,5}$

En marzo de 2019, esta enfermedad emergente fue declarada pandemia y se ha requerido una serie de modificaciones para llevar a cabo las actividades de la vida diaria. En el caso de los profesionales de la salud dedicados al tratamiento de la patología ortopédica hemos tenido que realizar modificaciones en nuestro quehacer diario para evitar el riesgo de contagio. Toda esta nueva información que se genera a partir de los países donde esta pandemia inició y donde se tiene mayor experiencia basada en la atención de este tipo de pacientes, además de que ha colapsado sus sistemas de salud, nos lleva a replantear la forma de atención de la manera que estábamos acostumbrados. 
Nos vemos en la necesidad de optimizar los recursos disponibles, siendo prioritaria la protección del personal sanitario. Se ha limitado la cirugía electiva y se redistribuye el acceso a los servicios de cuidados intensivos, lo cual modifica el acceso a los sistemas de salud. Se debe contar con el equipo de protección adecuado en todo procedimiento o evaluación realizada a los pacientes de acuerdo a los protocolos establecidos para cada caso. A pesar de que los cirujanos de pie y tobillo no tenemos un rol principal en la atención de pacientes con COVID-19, debemos seguir los lineamientos en la atención de pacientes en los que se puede postergar tanto la consulta externa como algunos procedimientos quirúrgicos. ${ }^{1,3-6}$

\section{ATENCIÓN DE PACIENTES POSTOPERADOS}

Incluye los pacientes operados seis meses previos al brote de COVID-19, para ellos hay que reducir el acceso a servicios de emergencia, posponer consultas no urgentes o hacer uso de la telemedicina. ${ }^{1,3,6-8}$

En todos los padecimientos ortopédicos que requieren atención ambulatoria o consulta se debe revisar un paciente a la vez, llevando a cabo todo el protocolo de sanitización del espacio entre cada paciente y dejar espacio para los pacientes postoperados. También se debe proponer la opción de realizar telemedicina o reprogramar la consulta.

\section{PROGRAMACIÓN DE CIRUGÍA ELECTIVA}

Aunque estamos en la fase III de esta pandemia, se deben optimizar las camas hospitalarias disponibles así como los recursos humanos, por lo que durante esta fase se recomienda no programar cirugía electiva, por otro lado se ha conseguido mantener los sistemas de salud estables en diferentes regiones, por lo que se tiene que empezar a planificar el retorno a las actividades normales en las siguientes semanas, esto incluye la cirugía programada en la que si es necesario se debe llevar a cabo en hospitales libres de COVID, respetando regulaciones locales y nacionales; durante esta fase de reorganización y reestructuración es necesario realizar filtros a pacientes y personal de salud descartando la presencia de fiebre o síntomas de resfriado o tos, incluso tener una prueba de COVID negativa teniendo en cuenta que el hospital puede llegar a ser un vector de infección. Se deben priorizar pacientes con alta

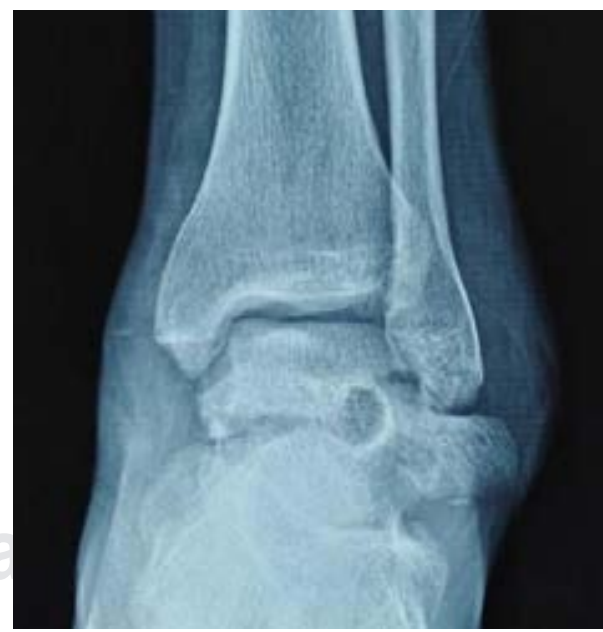

Figura 1: Radiografía AP de tobillo, masculino de 18 años, trauma de alta energía, fractura de astrágalo, cuello y fragmento lateral. 
discapacidad y ausencia de comorbilidad (evitar que requieran terapia intensiva). ${ }^{1,3-5,7-9}$

Por lo cual, los pacientes que ya tenían una cirugía programada, se debe valorar su suspensión, salvo la oncológica, o aquélla que si demora dejará secuelas. En la atención quirúrgica se debe preferir la anestesia regional en vez de la general, si es necesario llevar a cabo ésta, se debe considerar la intubación de secuencia rápida sin ventilación del paciente para evitar la aerolización y dispersión del virus en la sala de operaciones; se prefiere el uso de quirófanos con presión negativa neutra, evitar el uso de escafandras con recirculación

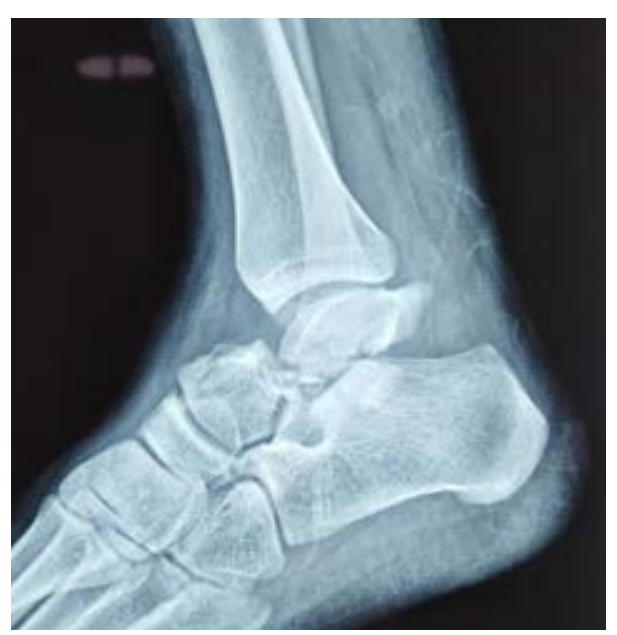

Figura 2: Radiografía lateral de tobillo. Se aprecia fractura en el cuello del astrágalo, desplazada. de aire; además de tener en cuenta e informar al paciente que existe un aumento de hasta $20 \%$ de mortalidad en pacientes portadores de COVID al realizar su cirugía electiva.

\section{TRAUMATOLOGÍA/EMERGENCIA}

Los pacientes con padecimientos ortopédicos de pie y tobillo que no deben suspender sus tratamientos durante la pandemia son aquéllos con trauma agudo (Figuras 1 y 2), fracturas expuestas, pacientes con síndrome compartamental, infección de heridas y pie diabético complicado.

Si requieren una inmovilización de la extremidad deberá considerarse la opción de colocar ortesis removibles con la finalidad de disminuir el número de consultas presenciales con el paciente.

Considerar cualquier intervención con alta posibilidad de contaminación, ya que se produce gran cantidad de material en forma de aerosoles y gotas de líquidos biológicos. También se debe considerar a todos los pacientes que no tengan una prueba negativa para COVID como potencialmente enfermos (durante el periodo de incubación del virus el paciente permanece asintomático), por lo cual deberán seguirse todas las indicaciones de protección (protección ocular completa, mascarilla N95, batas impermeables, protección completa para el calzado), principalmente si se utilizan equipos de poder durante el procedimiento. ${ }^{3-7}$

\section{CONCLUSIONES}

La enfermedad por COVID-19 vino a cambiar los hábitos de la vida cotidiana y del ejercicio de nuestra profesión, incluyendo nuestro rol como cirujanos de pie y tobillo. Actualmente nuestro trabajo debe estar enfocado en ser un ejemplo 
positivo en el seguimiento de las reglas establecidas para nuestra protección y la de los pacientes en beneficio de nuestra sociedad; tomando en cuenta una programación quirúrgica y un seguimiento apropiados para nuestros pacientes. También debemos prepararnos de una forma ordenada para cuando termine la emergencia sanitaria, pues se tendrá una alta demanda en la solicitud de atención en consulta y en cirugía, adquiriendo la capacidad para tomar decisiones en el discernimiento de aquellos pacientes que necesiten una atención prioritaria sin que ello vuelva a poner en riesgo nuestro sistema de salud.

\section{BIBLIOGRAFÍA}

1. Recomendaciones de la Sociedad Española de Cirugía Ortopédica y Traumatología frente al COVID-19. Disponible en: www.secot.es.

2. Kampf G, Todt D, Pfeander S, Steinmann E. Persistence of coronaviruses on inanimate surfaces and their inactivation with biological agents. J Hosp Infect. 2020; 104 (3): 246-251. Available in: https://doi. org/10.1016/j.jhin.2020.01.22.

3. Giuseppe U, D'Ambrosi R. Being a foot and ankle surgeon in Italy in the era of COVID-19. Knee Surg Sports Traumatol Arthrosc. 2020, 1-4.

4. COVID19-Evidence-Based Best Practice Guidelines Specific to Orthopaedic Surgeons. Available in: https://rcsi.com.

5. Montero FA, Maseda E, Adalia BR, Aguilar G, González CR, Gómez-Herreras Jl, et al. Recomendaciones prácticas para el manejo perioperatorio del paciente con sospecha o infección grave por Coronavirus SARS-CoV-2. Rev Esp Anestesiol Reanim. 2020; 67 (5): 253-260.

6. Shaoqing L, Fang J, Wating S, Chang C, Jingli C, Wei M, et al. Clinical characteristics and outcomes of patients undergoing surgeries during the incubation period of COVID-19 infection. Eclinical Medicine. 2020; 9: 41.

7. Clinical guide for management of trauma and orthopaedic patients during the coronavirus pandemic. NHS, 2020.

8. Balibrea JM, Badia JM, Rubio PI, Antona EM, Álvarez PE, García BS, et al. Manejo quirúrgico de pacientes con infección por COVID-19. Recomendaciones de la Asociación española de cirujanos. Cir Esp. 2020; 98 (5): 251-259. Disponible en https://doi.org/1016/j.ciresp2020.03.001.

9. COVID-19: Elective Case Triage Guidelines for Surgical Care. American College of Surgeons. Available in: https://wwwfacs.org/covid-19/clinical-guidance/elective-case. 